\title{
THE RELATION BETWEEN THE DRIVER BEHAVIOR QUESTIONNIARE, DEMOGRAPHICS, AND DRIVING HISTORY
}

\author{
Birsen Donmez ${ }^{1, *}$, Alea Mehler ${ }^{2}$, Joonbum Lee $^{2}$, Bruce Mehler $^{2}$, Bryan Reimer $^{2}$ \\ ${ }^{1}$ Dept. Mechanical \& Industrial Engineering, University of Toronto, Toronto, Canada \\ ${ }^{2}$ MIT AgeLab \& New England Univ. Transportation Center, Cambridge, Massachusetts, USA \\ Email: donmez@mie.utoronto.ca
}

\begin{abstract}
Summary: This paper presents an analysis of responses obtained on the Driver Behavior Questionnaire (DBQ) and self-reported history of the frequency of crashes, citations, and warnings in a sample of 562 drivers. The sample was closely balanced by gender and distributed in a broadly proportional manner across an age range of from 20 to 69 years. As has been previously reported, age and gender were found to be related to both DBQ scores and crash rates. The size and demographic distribution of the sample allowed an analysis to be run looking at the relationships of DBQ subscale scores with crashes, citations, and warnings, while controlling for age and gender. The results show that higher violation scores are positively associated with increases in self-reported crash and citation likelihoods; the less serious but apparently more common experience of receiving a warning for one's driving behavior has a significant positive association with both violation and lapse scores. The extent to which these findings can be considered relevant to the overall driving population is enhanced from previous research given the sample size and age/gender balance.
\end{abstract}

\section{INTRODUCTION}

Factors that may lead or contribute to vehicle crashes have been widely studied using various methods (e.g., Stutts et al., 2001; Dingus et al., 2006). One stream of research focuses on using objective measurements of driving performance under laboratory conditions, in field experiments, or in a naturalistic environment (e.g., Reimer, Mehler, Dobres, \& Coughlin, 2013; Klauer et al., 2006), while another stream focuses on subjective measurements of driving behaviour using surveys or questionnaires (e.g., Zhao et al., 2012; Chen et al., 2016). The present study focuses on the latter stream by exploring the relationships between self-reported driving history and self-reported driving behaviors while controlling for driver age and gender, factors that are significantly related to driver behavior and crash likelihood. Driving behaviors are assessed through the Driver Behaviour Questionnaire (DBQ), an instrument widely used in driving research (de Winter \& Dodou, 2010; Lee, Reimer, Mehler, \& Coughlin, 2016; Parker, McDonald, Rabbitt, \& Sutcliffe, 2000; Parker, West, Stradling, \& Manstead, 1995; Reimer et al., 2005; Stephens \& Groeger, 2009; Zhao et al., 2012; Zhao, Reimer, Mehler, D’Ambrosio, \& Coughlin, 2013). A U.S. version of the DBQ (see Reimer et al., 2005) is used, which consists of 24 questions and three sub-scales (Errors: misjudgements or failures of observation that could be hazardous to others, Lapses: absent-minded behaviors which may be frustrating or have negative consequences for the driver responsible, but generally do not pose a threat to anyone's safety, and Violations: deliberate contraventions of legally regulated or socially accepted behaviors associated with safe vehicle operation). 
Two main objectives of the present study are to: (a) investigate relations among DBQ, driving history, and demographic information, and (b) extend the investigation to predict crashes/citations/warnings using DBQ and demographic information. To achieve these goals, a total of 562 drivers' DBQ scores, demographics, and driving history information (e.g., crashes and citations) were collected and analyzed. The sample was closely balanced by gender and distributed in a broadly proportional manner across an age range of from 20 to 69 years. The size as well as the age and gender distribution of the present dataset allowed for an assessment of the extent to which relationships exist between DBQ scores and self-reported crash, citation, and warning histories while statistically controlling for age and gender effects, an approach that has not been possible with datasets reported by previous research. Age and gender have effects on both driver behaviours and crash risks (e.g., Tefft, 2012) and hence may mediate the relations of DBQ responses with crash, citation, and warning histories. These potential mediating effects are explored and controlled for through statistical modeling approaches utilized in this paper.

\section{METHODS}

Data were drawn from intake questionnaires collected in a series of on-road studies investigating voice-based secondary tasks conducted between 2012 and 2015 (Mehler et al. 2014; Mehler et al., 2015a, 2015b, 2015c; Mehler et al., 2016; Reimer et al. 2013; Reimer et al. 2014; Reimer et al., 2015). The studies required that participants attest during a recruitment screening to being active drivers (driving on average 3 or more times a week), not having been a driver in a police reported accident in the past year, and being in reasonably good health for their age.

\section{Questionnaires}

Along with DBQ, demographic information as well as driving history data were collected from each participant. Participants reported their age, gender, frequency of driving, and education level. They also reported the number of times they had received a warning, a citation/ticket, as well as the number of times they had been in a crash as a driver in the past five years. No independent verification of the accuracy of self-report was undertaken. There were no missing data for the sample analyzed.

\section{Participants}

The sample reported here consists of 562 participants (288 females, 274 males), aged 20-69 (female: $M=43, S D=17.3$; male: $M=41, S D=17.6$ ); this total is larger than the sum of the analysis samples reported for individual on-road studies as it includes participants who did not go on-road due to inability to learn tasks, were dropped due to poor weather or traffic conditions, had incomplete instrumented vehicle data, etc. Participants were drawn largely from eastern Massachusetts in the U.S., and were identified primarily using online and newspaper advertisements as well as social media including both physical and virtual bulletin board postings. Compensation ranged between $\$ 75$ and $\$ 150$ depending in part on the duration of the individual study. As was expected from the study recruitment criteria, all participants reported on average driving at least a few days a week. The respondents were in general well educated; only 23 participants were high-school graduates with no college education, 119 had some college 
education (67 of whom were 20-24 years old), 179 participants completed college as their highest degree, and the remaining 240 participants had at least some post-graduate education.

Table 1 presents the gender and age group breakdown generally in alignment with those suggested by NHTSA (2013), with the exception of the suggested 55+ group being split into two categories in order to better address the increasing prevalence of older drivers. Although the NHTSA age ranges were built mostly based on the age distribution of U.S. drivers, they also capture significant shifts in crash risk as driver age increases (Tefft, 2012).

Table 1. Sample age/gender distribution

\begin{tabular}{lllllll} 
& \multicolumn{6}{c}{ Age Groups } \\
\cline { 2 - 6 } Gender & $\mathbf{2 0 - 2 4}$ & $\mathbf{2 5 - 3 9}$ & $\mathbf{4 0 - 5 4}$ & $\mathbf{5 5 - 6 4}$ & $\mathbf{6 5 - 6 9}$ & Total \\
\hline Female & 66 & 67 & 53 & 64 & 38 & 288 \\
Male & 73 & 68 & 44 & 49 & 40 & 274 \\
\hline Total & 139 & 135 & 97 & 113 & 78 & 562
\end{tabular}

\section{RESULTS}

\section{Relations of Crashes/Citations/Warnings with Age and Gender}

The majority of participants reported to not having any crashes $(n=463)$, citations $(n=427)$, or warnings $(n=379)$ in the past five years. The breakdown based on age and gender is reported in Table 2. In particular, the percentage of the response frequency is reported with the denominator of total number of female (or male) respondents within each age group. For example, the first entry in the table is $73 \%$, which represents the percentage of female participants between 20-24 years old who reported to having no crashes in the past five years; $27 \%(n=18)$ of this group $(n$ =66) reported having at least one crash.

Table 2. Crash, citation, warning responses across age and gender

\begin{tabular}{|c|c|c|c|c|c|c|c|c|c|c|c|}
\hline & \multicolumn{10}{|c|}{ Age Group } \\
\hline & & \multicolumn{2}{|c|}{$20-24$} & \multicolumn{2}{|c|}{ 25-39 } & \multicolumn{2}{|c|}{$40-54$} & \multicolumn{2}{|c|}{$55-64$} & \multicolumn{2}{|c|}{ 65-69 } \\
\hline & & $\mathbf{F}$ & M & $\mathbf{F}$ & M & $\mathbf{F}$ & M & $\mathbf{F}$ & M & $\mathbf{F}$ & $\mathbf{M}$ \\
\hline & & $n=66$ & 73 & 77 & 68 & 53 & 44 & 64 & 49 & 38 & 40 \\
\hline \multirow[t]{2}{*}{ Crashes } & $0 \quad(n=463)$ & $73 \%$ & $70 \%$ & $88 \%$ & $88 \%$ & $89 \%$ & $86 \%$ & $80 \%$ & $86 \%$ & $84 \%$ & $88 \%$ \\
\hline & $\geq 1(n=99)$ & $27 \%$ & $30 \%$ & $12 \%$ & $12 \%$ & $11 \%$ & $14 \%$ & $20 \%$ & $14 \%$ & $16 \%$ & $12 \%$ \\
\hline \multirow[t]{2}{*}{ Citations } & $0 \quad(n=427)$ & $70 \%$ & $51 \%$ & $88 \%$ & $69 \%$ & $83 \%$ & $73 \%$ & $91 \%$ & $86 \%$ & $76 \%$ & $83 \%$ \\
\hline & $\geq 1(n=135)$ & $30 \%$ & $49 \%$ & $12 \%$ & $31 \%$ & $17 \%$ & $27 \%$ & $9 \%$ & $14 \%$ & $24 \%$ & $17 \%$ \\
\hline \multirow[t]{2}{*}{ Warnings } & $0 \quad(n=379)$ & $65 \%$ & $52 \%$ & $76 \%$ & $68 \%$ & $72 \%$ & $73 \%$ & $73 \%$ & $63 \%$ & $63 \%$ & $73 \%$ \\
\hline & $\geq 1(n=183)$ & $35 \%$ & $48 \%$ & $24 \%$ & $32 \%$ & $28 \%$ & $27 \%$ & $27 \%$ & $37 \%$ & $37 \%$ & $27 \%$ \\
\hline
\end{tabular}

Logistic regression models were built on self-reported crash involvement, citations, and warnings with age, gender, and their interaction as explanatory variables. Only age-group had a significant effect on self-reported crash likelihood, $\chi^{2}(4)=16.57, p=.002$. Follow up contrasts revealed that 20-24 year olds had higher odds of a crash compared to all other age groups. Both age-group, $\chi^{2}(4)=27.16, p=<.0001$, and gender, $\chi^{2}(1)=5.81, p=.02$, had significant main 
effects on citation likelihood. 20-24 year olds again had higher odds compared to all other age groups. Further, males were more likely to report receiving citations than females. No significant effects were found for warnings.

\section{Relations of DBQ with Age and Gender}

Scores for the three DBQ subscales of errors, lapses, and violations are presented in Table 3. The three subscales were significantly correlated, with correlation coefficients around 0.5 between errors and other subscales (lapses: $r=0.56, p<.0001$; violations: $r=0.51, p<.0001$ ), and at 0.37 between lapses and violations $(r=0.37, p<.0001)$.

Separate regression models were built to investigate whether age and gender had an effect on these three subscales. There was a significant age effect for violations, $F(4,552)=3.33, p=.01$, and significant gender effects for lapses, $F(1,552)=5.38, p=.02$, and errors, $F(1,552)=9.91, p$ $=.002$. Follow-up contrasts revealed that 20-24 year olds had higher scores for violations compared to all other age groups but 40-54 year olds, and females had higher scores for lapses and errors compared to males.

Table 3. DBQ responses across age and gender $(M=$ mean, $S D=$ standard deviation)

\begin{tabular}{|c|c|c|c|c|c|c|c|c|c|c|}
\hline & \multicolumn{10}{|c|}{ Age Group } \\
\hline & \multicolumn{2}{|c|}{$20-24$} & \multicolumn{2}{|c|}{ 25-39 } & \multicolumn{2}{|c|}{$40-54$} & \multicolumn{2}{|c|}{$55-64$} & \multicolumn{2}{|c|}{ 65-69 } \\
\hline & $\mathbf{F}$ & $\mathbf{M}$ & $\mathbf{F}$ & $\mathbf{M}$ & $\mathbf{F}$ & M & $\mathbf{F}$ & $\mathbf{M}$ & $\mathbf{F}$ & $\mathbf{M}$ \\
\hline \multirow[t]{2}{*}{ Errors } & M: 5.71 & 4.78 & 5.34 & 5.16 & 5.49 & 3.75 & 4.36 & 4.69 & 6.61 & 3.5 \\
\hline & SD: 3.53 & 2.88 & 3.63 & 3.54 & 3.83 & 3.59 & 3.12 & 3.55 & 3.47 & 2.60 \\
\hline \multirow[t]{2}{*}{ Lapses } & M: 6.85 & 6.06 & 6.69 & 6.43 & 7.23 & 6.52 & 6.93 & 6.31 & 7.03 & 5.68 \\
\hline & SD: 3.50 & 4.27 & 3.73 & 3.25 & 4.17 & 4.28 & 3.93 & 3.36 & 4.26 & 2.62 \\
\hline \multirow[t]{2}{*}{ Violations } & M: 6.85 & 8.34 & 5.85 & 6.96 & 7.25 & 6.32 & 6.12 & 6.39 & 5.63 & 5.75 \\
\hline & SD: 3.92 & 5.09 & 3.42 & 4.60 & 4.26 & 4.85 & 3.67 & 4.12 & 2.96 & 2.57 \\
\hline
\end{tabular}

\section{Predicting Crashes/Citations/Warnings with DBQ while controlling for Age and Gender}

Given that age and gender relate to both self-reported crash/citation/warning history and DBQ subscales, the relation between DBQ and self-reported history should be assessed while controlling for age and gender effects.

Logistic regression models were built on self-reported crash involvement, citations, and warnings with the three DBQ subscales as well as age, gender, and their interaction as explanatory variables. For crash involvement and citations, the only significant DBQ subscale was violations: $\chi^{2}(1)=4.36, p=.04$ and $\chi^{2}(1)=12.86, p=.0003$, respectively. An increase in the violation score was associated with increases in self-reported crash and citation likelihoods. For warnings, all three subscales had an effect, errors: $\chi^{2}(1)=7.86, p=.005$, lapses: $\chi^{2}(1)=12.27, p$ $=.0005$, and violations: $\chi^{2}(1)=15.34, p<.0001$. Increases in the violation and lapse scores were associated with an increase in the likelihood of warnings, whereas an increase in the error score was associated with a decrease. 
The age and gender effects obtained through the models that included DBQ were similar to the effects reported earlier for the models that did not include DBQ. In particular, age group was significant for violations, $\chi^{2}(4)=13.98, p=.007$, with the 20-24 year-olds having a higher likelihood of self-reported crash involvement. Both age-group, $\chi^{2}(4)=21.56, p=.0002$, and gender, $\chi^{2}(1)=5.35, p=.02$, had significant main effects on citation likelihood, with the 20-24 year olds again having higher odds than other age groups and males having higher odds than females. No age or gender effect was found for warnings.

\section{DISCUSSION}

The finding that DBQ violations scores are associated with increased odds of having been a driver in a crash, and errors and lapses scores showing lower or non-significant relationships, is in line with previous findings (see review in Zhao et al. 2012). Similarly, it has previously been reported that age and gender tend to be related to DBQ scores as well as with crash rates; younger drivers and males tend to have higher DBQ violation scores, whereas women and older drivers tend to show higher error scores (Kontogiannis, et al., 2002; Reason, et al., 1990). Thus, as Zhao et al. (2012) noted, a consideration of the distribution of these demographic factors is likely important in interpreting findings from specific datasets and may partially explain some reports of contradictory or non-significant relationships. This highlights the value of reference to a broad age distribution and a relatively well-balanced gender sample when the goal is to develop an understanding of relationships across the driving population.

The size as well as the age and gender distribution of the present dataset allowed for an assessment of the extent to which relationships exist between DBQ scores and self-reported crash, citation, and warning history while statistically controlling for age and gender effects. The results show that higher violations scores are positively associated with increases in self-reported crash and citation likelihood. It can further be observed that the less serious but apparently more common experience of receiving a warning for one's driving behavior shows a significant positive association with both violation and lapse scores. It is interesting to note that an inverse relationship appears between error scores and the likelihood of having received a warning; further research is needed to investigate this finding.

A limitation of the sample analyzed in this paper is the exclusion of drivers who reported to being a driver in a police reported crash in the year immediately prior to the study. This exclusion was necessary as a safety precaution for conducting the on-road studies that followed the questionnaire data collection. Along with this exclusion criterion, the participants being recruited from eastern Massachusetts area on a voluntary basis lead to a sample that may not be representative of the general driving population. Further, there are certain limitations and possible biases associated with self-reported data. It is possible that the participants may not have been able to accurately recall their crash, violation, and warning histories. It is also possible that their questionnaire responses may have been affected from a social desirability bias; however, previous analysis on a subset of this dataset reported in Zhao et al. (2012) revealed significant relations between DBQ scores and on-road driving behaviours in directions that are expected (e.g., higher violations scores were correlated with higher speeds maintained). To minimize these potential limitations and biases, future research should incorporate ways to cross-check recall data with actual driver records and ways to induce truthfulness for DBQ responses. 


\section{ACNKOWLEDGEMENTS}

Support for this work was provided by the US DOT's Region I New England University Transportation Center at MIT and the Toyota Class Action Settlement Safety Research and Education Program. The views and conclusions being expressed are those of the authors, and have not been sponsored, approved, or endorsed by Toyota or plaintiffs' class counsel.

\section{REFERENCES}

Chen, H-Y. W., Donmez, B., Hoekstra-Atwood, L., \& Marulanda, S. (2016). Self-reported engagement in driver distraction: An application of the Theory of Planned Behaviour. Transportation Research Part F: Traffic Psychology and Behaviour, 38, 151-163.

de Winter, J. C. F., \& Dodou, D. (2010). The driver behaviour questionnaire as a predictor of accidents: a meta-analysis. Journal of Safety Research, 41, 463-470.

Dingus, T. A., Klauer, S. G., Neale, V. L., Petersen, A., Lee, S. E., Sudweeks, J. D., et al. (2006). The 100-car Naturalistic Driving Study. Phase II-Results of the 100-car Field Experiment (No. DOT HS 810 593). U.S. Department of Transportation.

Klauer, S. G., Dingus, T. A., Neale, V. L., Sudweeks, J. D., \& Ramsey, D. J. (2006). The Impact of Driver Inattention on Near-Crash/Crash Risk: An Analysis using the 100-Car Naturalistic Driving Study Data (No. DOT HS 810 594). U.S. Department of Transportation.

Kontogiannis, T., Kossiavelou, Z., \& Marmaras, N. (2002). Self-reports of aberrant behavior on the roads: Errors and violations in a sample of Greek drivers. Accident Analysis \& Prevention, 34(3), 2002.

Lee, J., Reimer, B., Mehler, B., \& Coughlin, J.F. (2016). Sensation seeking and drivers' glance behavior while engaging in a secondary task. Proceedings of the Human Factors and Ergonomics Society Annual Meeting, Washington D.C.

Mehler, B., Kidd, D., Reimer, B., Reagan, I., Dobres, J. \& McCartt, A. (2016). Multi-modal assessment of on-road demand of voice and manual phone calling and voice navigation entry across two embedded vehicle systems. Ergonomics, 59(3), 344-367.

Mehler, B., Reimer, B., Dobres, J., \& Coughlin, J.F. (2015a). Assessing the Demands of Voice Based In-Vehicle Interfaces - Phase II Experiment 3 - 2015 Toyota Corolla. MIT AgeLab Technical Report 2015-14. MIT, Cambridge, MA.

Mehler, B., Reimer, B., Dobres, J., McAnulty, H., \& Coughlin, J.F. (2015b). Assessing the Demands of Voice Based In-Vehicle Interfaces - Phase II Experiment 1 - 2014 Chevrolet Impala. MIT AgeLab Technical Report 2015-6A. MIT, Cambridge, MA.

Mehler, B., Reimer, B., Dobres, J., McAnulty, H., Mehler, A., Munger, D., \& Coughlin, J.F. (2014). Further Evaluation of the Effects of a Production Level "Voice-Command" Interface on Driver Behavior: Replication and a Consideration of the Significance of Training Method. MIT AgeLab Technical Report 2014-2. MIT, Cambridge, MA.

Mehler, B., Reimer, B., McAnulty, H., Dobres, J., Lee, J. \& Coughlin, J.F. (2015c). Assessing the Demands of Voice Based In-Vehicle Interfaces - Phase II Experiment 2 - 2014 Mercedes CLA. MIT AgeLab Technical Report 2015-8. MIT, Cambridge, MA. 
National Highway Traffic Safety Administration (2013). Visual-Manual NHTSA Driver Distraction Guidelines for In-Vehicle Electronic Devices (No. NHTSA-2010-0053). U.S. Department of Transportation, Washington, DC.

Parker, D., McDonald, L., Rabbitt, P., \& Sutcliffe, P. (2000). Elderly drivers and their accidents: The aging driver questionnaire. Accident Analysis \& Prevention, 32, 751-759.

Parker, D., West, R., Stradling, S. G., \& Manstead, A. S. R. (1995). Behavioural characteristics and involvement in different types of traffic accident. Accident Analysis \& Prevention, 27(4), 571-581.

Reason, J., Manstead, A., Stradling, S., Baxter, J., \& Campbell, K. (1990). Errors and violations on the roads: A real distinction? Ergonomics, 33(10), 1315-1332.

Reimer, B., D'Ambrosio, L. A., Gilbert, J., Coughlin, J. F., Biederman, J., Surman, C., et al. (2005). Behavior differences in drivers with Attention Deficit Hyperactivity Disorder: The Driving Behavior Questionnaire. Accident Analysis \& Prevention, 37(6), 996-1004.

Reimer, B., Mehler, B., Dobres, J. \& Coughlin, J.F. (2013). The Effects of a Production Level "Voice-Command" Interface on Driver Behavior: Reported Workload, Physiology, Visual Attention, and Driving Performance. MIT AgeLab Technical Report 2013-17A. MIT, Cambridge, MA.

Reimer, B., Mehler, B., Dobres, J., \& Coughlin, J.F. (2015). Assessing the Demands of Voice Based In-Vehicle Interfaces - Phase II Experiment 4 - An Exploratory Study of Driver Behavior With and Without Assistive Cruise Control (ACC). MIT AgeLab Technical Report 2015-15. MIT, Cambridge, MA.

Reimer, B., Mehler, B., Dobres, J., McAnulty, H., Mehler, A., Munger, D., \& Rumpold, A. (2014). Effects of an 'expert mode' voice command system on task performance, glance behavior $\&$ driver physiology. Proceedings of the $6^{\text {th }}$ International Conference on Automotive User Interfaces and Interactive Vehicular Applications, Seattle, WA.

Stephens, A. N., \& Groeger, J. A. (2009). Situational specificity of trait influences on drivers' evaluations and driving behavior. Transportation Research Part F: Traffic Psychology and Behaviour, 12(1), 29-39.

Stutts, J., Reinfurt, D., Staplin, L., \& Rodgman, E. (2012). The Role of Driver Distraction in Traffic Crashes. Washington, D.C.: AAA Foundation for Traffic Safety.

Tefft, B. C. (2012). Motor Vehicle Crashes, Injuries, and Deaths in Relation to Driver Age: United States, 1995-2010. Washington, D.C.: AAA Foundation for Traffic Safety.

Zhao, N., Mehler, B., Reimer, B., D’Ambrosio, L.A., Mehler, A., \& Coughlin, J.F. (2012). An investigation of the relationship between the Driving Behavior Questionnaire and objective measures of highway driving behavior. Transportation Research Part F: Traffic Psychology and Behavior, 15(1), 676-685.

Zhao, N., Reimer, B., Mehler, B., D’Ambrosio, L.A., \& Coughlin, J.F. (2013). Self-reported and observed risky driving behaviors among frequent and infrequent cell phone users. Accident Analysis \& Prevention, 61, 71-77. 